\title{
THE MACKEY OBSTRUCTION AND THE COADJOINT ORBITS
}

\author{
ZONGYI LI
}

\begin{abstract}
This paper studies the Mackey obstruction representation theory at the coadjoint orbit level. It shows how to get rid of such obstructions and to get orbits of the "little groups". Such little group data is essential for inductive construction of coadjoint orbits of general Lie groups.
\end{abstract}

\section{INTRODUCTION}

Let $G$ be a real Lie group, $N \subset G$ be a normal subgroup, and $N^{\wedge}$ be the set of equivalent classes of irreducible unitary representations of $N$. Since $N$ is normal in $G$, there is a natural action of $G$ on $N^{\wedge}$. Pick a representation $\rho \in N^{\wedge}$. We assume that $G$ fixes $\rho$. One would like to know if $\rho$ can be extended to an irreducible unitary representation of $G$ in the sense that there is such a unitary representation of $G$ whose restriction to $N$ is $\rho$. The answer in general is no. There is an obstruction (called the Mackey obstruction) to extending $\rho$ to a unitary representation of $G$. Based on the quantization method, one can relate representations to the coadjoint orbits of groups. For example, there exists a one-to-one correspondence between the coadjoint orbits and the irreducible unitary representations for the nilpotent groups (see [5]) and the solvable groups (see [1]). Therefore, it will help us a lot to understand the representation picture if we can understand the corresponding problems on coadjoint orbits; in other words, at the symplectic geometry level. The Mackey obstruction can be interpreted as the obstruction to realizing the coadjoint orbit $Y$ of $N$ as a coadjoint orbit of $G$ under the assumption that the $G$-action stabilizes $Y$. We prove in this paper that in the case that $G$ fixes $Y$, this orbit is always a coadjoint orbit of some central extension $G^{\sigma}$ of $G$ with respect to the circle group $T$. To get a coadjoint orbit from this orbit $Y$, we need to find another coadjoint orbit $W$ of some group extension $G^{-\sigma}$ which is dual to our first central extension $G^{\sigma}$. Then the product space $Y \times W$ will be a coadjoint orbit of $G$ while the obstructions cancel out. This treatment reflects Auslander-

Received by the editors May 1, 1994.

1991 Mathematics Subject Classification. Primary 53C15.

Key words and phrases. Coadjoint orbit, the Mackey obstruction, symplectic manifold, Hamiltonian action, moment map.

Research partially supported by DOE Grant DE-FG02-88ER25066. 
Kostant's treatment of the Mackey obstruction in their representation theory of solvable Lie groups in [1].

\section{ACKNOWLEDGMENTS}

I am deeply grateful to Professor V. Guillemin for introducing me to this subject. Many thanks to Professor Guillemin and Joshua Sher for reading the whole manuscript carefully and making some important corrections. Thanks also to Professor B. Kostant for suggesting this topic.

\section{The MACKey OBSTRUCTION AND PROJECTIVE REPRESENTATIONS}

Let us review the problems associated with extending the representation $\rho \in$ $N^{\wedge}$ to $G$ under the assumption that $G$ fixes $\rho$, and then look at the Mackey obstruction. In general, $\rho$ can only extend to a projective representation $\rho^{\sigma}$ of $G$. Namely,

$$
\rho^{\sigma}(a b)=\sigma(a, b) \rho^{\sigma}(a) \rho^{\sigma}(b)
$$

for any $a, b \in G$, where the 2-cocycle $\sigma$ satisfies

$$
\begin{gathered}
\sigma: G \times G \rightarrow \mathbb{C}^{\times}, \quad|\sigma(a, b)|=1, \\
\sigma(e, a)=\sigma(a, e)=1,
\end{gathered}
$$

and

$$
\sigma(a b, c) \sigma(a, b)=\sigma(a, b c) \sigma(b, c),
$$

where $e$ is the identity of $G$. Now $\rho^{\sigma}$ is a representation of $G$ if $\sigma \equiv 1$. But in any case $\rho$ extends to a representation of some central extension $G^{\sigma}$ of $G$ with respect to the circle group $T$. We define $G^{\sigma}=\{(t, a) \in T \times G\}$, where the multiplication of this group is defined as

$$
(t, a) \cdot(s, b)=(t s / \sigma(a, b), a b) .
$$

Then $G^{\sigma}$ is a group with the identity $(1, e)$ and $\left(\sigma\left(a, a^{-1}\right) / t, a^{-1}\right)$ is the inverse of $(t, a)$. We have the exact sequence of groups

$$
1 \rightarrow T \stackrel{i}{\longrightarrow} G^{\sigma} \stackrel{j}{\longrightarrow} G \longrightarrow e,
$$

where $i(t)=(t, e), j(t, a)=a$. We define

$$
\rho_{0}^{\sigma}: G^{\sigma} \rightarrow \operatorname{Aut}(\mathscr{H}),
$$

where $\mathscr{H}$ is the representation space of $\rho$, by

$$
\rho_{0}^{\sigma}(t, a)=t \rho^{\sigma}(a) \text {. }
$$

It is easy to check that $\rho_{0}^{\sigma}$ is a unitary representation of $G^{\sigma}$. The 2-cocycle $\sigma$ is the obstruction to extending $\rho$ to a representation of $G$. We may define the Mackey obstruction by the following procedure.

Let

(1) $1 \rightarrow Q \rightarrow E_{1} \stackrel{\phi_{1}}{\longrightarrow} F \rightarrow 1$ and

(2) $1 \rightarrow Z \rightarrow E_{2} \stackrel{\phi_{2}}{\longrightarrow} F \rightarrow 1$

be two exact sequences of groups where $Z$ is in the center of $Q$. Let $R \subset$ $E_{1} \times E_{2}$ consist of all pairs $\left(e_{1}, e_{2}\right) \in E_{1} \times E_{2}$ such that $\phi_{1}\left(e_{1}\right)=\phi_{2}\left(e_{2}\right)$. Let $S$ 
be the subgroup of $R$ of the form $\left(z, z^{-1}\right), z \in Z$. Define the Baer product $E_{1} \otimes E_{2}=R / S$. Note that if we define

$$
\phi\left(e_{1}, e_{2}\right)=\phi_{1}\left(e_{1}\right)=\phi_{2}\left(e_{2}\right), \quad\left(e_{1}, e_{2}\right) \in R,
$$

then $\phi$ defines a homomorphism of $E_{1} \otimes E_{2}$ onto $F$ with the kernel $(Q \times Z) / S$. Also, $(Q \times Z) / S$ is isomorphic to $Q$, so $E_{1} \otimes E_{2}$ satisfies the exact sequence

(3) $1 \rightarrow Q \rightarrow E_{1} \otimes E_{2} \rightarrow F \rightarrow 1$.

We call (3) the Baer product of (1) and (2).

For $\rho \in N^{\wedge}$, we define a unitary representation $\tilde{\rho}$ of $T \times N$ as follows:

$$
\tilde{\rho}(t v)=t \rho(v), \quad \text { for any } t \in T, v \in N \text {. }
$$

We have

Theorem 2.1 (Mackey). Given the group extension

(i) $1 \rightarrow T \times N \rightarrow T \times G \rightarrow G / N \rightarrow 1$,

there exists a unique group extension

(ii) $1 \rightarrow T \rightarrow F \rightarrow G / N \rightarrow 1$

satisfying the following conditions:

(A) $T$ is central in $F$;

(B) if $G^{\natural}=F \otimes(T \times G)$ is the Baer product, then there exists a unitary representation $\rho^{\natural}$ of $G^{\natural}$ such that $\left.\rho^{\natural}\right|_{T \times N}=\tilde{\rho}$.

Definition 2.1. The group extension (2) above is called the Mackey obstruction of $G$ at $\rho$ or the obstruction to extending $\rho$ from $N$ to $G$.

In the case that the exact sequence (2) is trivial, or splitting, Theorem 2.1 says that $\rho$ can be extended to a representation of $G$.

Remark 2.1. Auslander-Kostant and Brezin showed in [1] and [2] how to compute the Mackey obstruction from a particular 2-cocycle on Lie algebras in certain cases. Roughly, let $\mathfrak{g}$ and $\mathfrak{n}$ be the Lie algebras of $G$ and $N$, respectively. There is a subspace $\mathfrak{a}$ of $\mathfrak{g}$ such that

$$
\mathfrak{g}=\mathfrak{a} \oplus \mathfrak{n} \text { as vector spaces, and }\left.\beta\right|_{[\mathfrak{a}, \mathfrak{n}]}=0 .
$$

where $\beta$ is some element in $\mathfrak{g}^{*}$, the dual space of $\mathfrak{g}$. Then let $\tau: \mathfrak{g} \rightarrow \mathfrak{g} / \mathfrak{n}$ and let $\theta: \mathfrak{g} / \mathfrak{n} \rightarrow \mathfrak{a}$ be the unique linear mapping such that $\tau \circ \theta$ is the identity mapping of $\mathfrak{g} / \mathfrak{n}$. Define a bilinear form $\sigma$ on $\mathfrak{g} / \mathfrak{n}$ by

$$
\sigma(\bar{\xi}, \bar{\eta})=-\beta([\theta \bar{\xi}, \theta \bar{\eta}]), \quad \bar{\xi}, \bar{\eta} \in \mathfrak{g} / \mathfrak{n} .
$$

$\sigma$ gives rise to a 2-cocycle on $\mathfrak{g}$. It was also used to construct the Mackey obstruction in the articles mentioned above. We call it the Mackey 2-cocycle.

\section{SYMPLECTIC INDUCTION AND HOMOGENEOUS $G$-SPACES}

In order to discuss the Mackey obstruction at the orbit level, we need to review symplectic induction. Let $(M, \omega)$ be a symplectic manifold and $G$ a Lie group acting on $M$. This action is said to be symplectic if any element of $G$ gives rise to a symplectomorphism from $M$ to itself. Let $\mathfrak{g}=\operatorname{Lie}(G)$, the Lie algebra of $G$. For any $\xi \in \mathfrak{g}, x \in M$, the infinitesimal generator at $x$ related to $\xi$ is defined as

$$
\xi^{\sharp}(x)=\xi_{M}^{\sharp}(x)=\left.\frac{d}{d t}\right|_{t=0}(\exp (-t \xi) \cdot x) .
$$


Then the map $\xi \longmapsto \xi^{\sharp}$ gives rise to a Lie algebra homomorphism from $\mathfrak{g}$ to the Lie algebra of all vector fields on $M$, denoted by $\mathscr{X}(M)$. Let Poisson $(M)$ be $C^{\infty}(M)$ equipped with the Poisson bracket. If all $\xi^{\sharp}$ are Hamiltonian vector fields so that we have the lifting of the infinitesimal action

$$
\phi: \mathfrak{g} \longrightarrow \operatorname{Poisson}(M)
$$

such that the interior product $l\left(\xi^{\sharp}\right) \omega=d \phi(\xi)$ for any $\xi \in \mathfrak{g}$, and $\phi$ is a Lie algebra homomorphism, we say that the $G$-action on $M$ is Hamiltonian. In this case, we have the moment map $\Phi: M \longrightarrow \mathfrak{g}^{*}$ which is defined by

$$
\langle\Phi(x), \xi\rangle=\phi^{\xi}(x),
$$

where $\phi^{\xi}=\phi(\xi)$. $\Phi$ is $G$-equivariant with respect to the coadjoint action of $G$ on $\mathfrak{g}^{*}$.

Theorem 3.1 (Marsden-Weinstein). If $\alpha$ is a regular value of the moment map $\Phi$ and $B=\Phi^{-1}(\alpha) / G_{\alpha}$ is a well-defined manifold where $G_{\alpha}$ is the stabilizer subgroup of $\alpha \in \mathfrak{g}^{*}$, then $B=\Phi^{-1}(\alpha) / G_{\alpha}$ is a symplectic manifold with the symplectic form $\omega_{B}$ satisfying $i^{*} \omega=j^{*} \omega_{B}$ where $i: \Phi^{-1}(\alpha) \rightarrow M$ is the inclusion map and $j: \Phi^{-1}(\alpha) \rightarrow B$ is the projection.

If $X$ is a coadjoint orbit of $G$, at each $x \in X$, since any vector in $T_{x} X$ can be expressed as $\xi_{X}^{\sharp}(x)$ for some $\xi \in \mathfrak{g}$, there is a natural surjection from $\mathfrak{g}$ to $T_{x} X$. This gives us a canonical symplectic form $\omega_{X}$ on $X$. For any $x \in X$, $\xi, \eta \in \mathfrak{g}$, we define

$$
\omega_{X}\left(\xi_{X}^{\sharp}(x), \eta_{X}^{\sharp}(x)\right)(x)=-\langle x,[\xi, \eta]\rangle .
$$

It is easy to see that this definition only depends on $\xi_{X}^{\sharp}(x)$ and $\eta_{X}^{\sharp}(x)$. The moment map for the coadjoint action of $G$ on $\left(X, \omega_{X}\right)$ is just the inclusion. Kostant (also Souriau) proved (see [6])

Theorem 3.2. If $(M, \omega)$ is a Hamiltonian G-homogeneous space, the moment map is a covering map from $M$ to a coadjoint orbit of $G$.

\section{THE ORBIT Version OF the Mackey obstruction}

We now translate the Mackey obstruction into the language of symplectic geometry. Let $G$ and $N$ be as above with $N$ connected. Let $Y \subset \mathfrak{n}^{*}$ be a coadjoint orbit of $N$ such that the natural action of $G$ on $\mathfrak{n}^{*}$ stabilizes $Y$. We assume that the stabilizer subgroup $N_{p}$ of the coadjoint action of $N$ at some point $p$, hence all points, on $Y$ is connected. The Mackey obstruction can be interpreted as the obstruction to realizing $Y$ as a coadjoint orbit of $G$.

Proposition 4.1. The $G$-action on $Y$ is symplectic.

Proof. For any automorphism $\phi: \mathfrak{n} \rightarrow \mathfrak{n}, \phi^{t}$ preserves the Poisson structure on $\mathrm{n}^{*}$ so it carries symplectic leaves into symplectic leaves. Moreover, if $Y_{1}$ is a symplectic leaf and $Y_{2}=\phi^{t}\left(Y_{1}\right)$ then $\phi^{t}$ is a symplectomorphism of $Y_{1}$ onto $Y_{2}$.

Let $\Omega_{Y}$ be the canonical symplectic form on $Y$. Proposition 4.1 says that $l\left(\xi^{\sharp}\right) \Omega_{Y}$ is closed. In addition, if $N$ is simply connected it is easy to see that $Y$ 
is simply connected and hence $l\left(\xi^{\sharp}\right) \Omega_{Y}$ is exact. Indeed, $N \rightarrow Y$ is a fibration with fiber $N_{p}$. By the long exact sequence in homotopy

$$
\cdots \rightarrow \pi_{1}(N) \rightarrow \pi_{1}(Y) \rightarrow \pi_{0}\left(N_{p}\right) \rightarrow \pi_{0}(N),
$$

we see that if $\pi_{1}(N)=\pi_{0}(N)=0$, then $\pi_{1}(Y)=\pi_{0}\left(N_{p}\right)$. In other words, if $N$ is simply connected, then $N_{p}$ is connected if and only if $Y$ is simply connected. We will however show that $l\left(\xi^{\sharp}\right) \Omega_{Y}$ is exact even when $N$ is not simply connected and, in fact, we will construct a canonical function $\phi^{\xi}: Y \rightarrow$ $\mathbb{R}$ such that $l\left(\xi^{\sharp}\right) \Omega_{Y}=d \phi^{\xi}$. Pick a point $p_{0} \in Y$. We can write

$$
Y=\left\{v \cdot p_{0} ; v \in N\right\} \text {. }
$$

Let $x_{0}$ be an element in $\mathfrak{g}^{*}$ such that $\left.x_{0}\right|_{\mathfrak{n}}=p_{0}$. Define the map $\phi^{\xi}: Y \longrightarrow \mathbb{R}$ by

$$
\phi^{\xi}\left(v \cdot p_{0}\right)=\left\langle v \cdot x_{0}, \xi\right\rangle .
$$

From the following general lemma we see that it is well defined.

Lemma 4.1. Let $G$ be an arbitrary Lie group, $N \subset G$ a normal subgroup, and $Y$ a coadjoint orbit of $N$. Let

$$
K=G_{Y}=\{g \in G ; g \cdot q \in Y \text { for all } q \in Y\}
$$

be the stabilizer subgroup at $Y$, and $\mathfrak{k}=\operatorname{Lie}(K)$. Then for any $x \in \mathfrak{g}^{*}$ such that $\left.x\right|_{\mathfrak{n}}=p \in Y$,

$$
N_{p}^{0} \cdot x=x+\mathfrak{k}^{\perp}
$$

where $N_{p}^{0}$ is the connected component of the stabilizer subgroup $N_{p}$, and

$$
\mathfrak{k}^{\perp}=\left\{\beta \in \mathfrak{g}^{*},\left.\beta\right|_{\mathfrak{k}}=0\right\}
$$

is the annihilator of $\mathfrak{k}$.

Proof. Let $\mathfrak{n}_{p}=\operatorname{Lie}\left(N_{p}\right)$. We first look at the behavior of the infinitesimal action. Define

$$
j: \mathfrak{n}_{p} \longrightarrow \mathfrak{g}^{*}, \quad j(\xi)=\xi \cdot x
$$

for any $\xi \in \mathfrak{n}_{p}$; the right-hand side of (4-2) is the infinitesimal action of $\xi$ on $x$. Notice that

$$
\operatorname{Im}(j) \subseteq \mathfrak{k}^{\perp}
$$

Indeed, one can easily get

$$
\mathfrak{k}=\mathfrak{g}_{p}+\mathfrak{n} .
$$

Hence for any $\xi \in \mathfrak{n}_{p}, \eta=\eta_{1}+\eta_{2} \in \mathfrak{k}$, where $\eta_{1} \in \mathfrak{g}_{p}, \eta_{2} \in \mathfrak{n}$, and

$$
\langle\xi \cdot x, \eta\rangle=\left\langle\xi \cdot x, \eta_{1}\right\rangle+\left\langle\xi \cdot x, \eta_{2}\right\rangle \text {. }
$$

But

$\left\langle\xi \cdot x, \eta_{1}\right\rangle_{\mathfrak{g}}=-\left\langle x,\left[\xi, \eta_{1}\right]\right\rangle_{\mathfrak{g}}=\left\langle x,\left[\eta_{1}, \xi\right]\right\rangle_{\mathfrak{g}}=\left\langle p,\left[\eta_{1}, \xi\right]\right\rangle_{\mathfrak{n}}=-\left\langle\eta_{1} \cdot p, \xi\right\rangle_{\mathfrak{n}}=0$,

and

$$
\left\langle\xi \cdot x, \eta_{2}\right\rangle_{\mathfrak{g}}=-\left\langle x,\left[\xi, \eta_{2}\right]\right\rangle_{\mathfrak{g}}=-\left\langle p,\left[\xi, \eta_{2}\right]\right\rangle_{\mathfrak{n}}=-\left\langle\xi \cdot p, \eta_{2}\right\rangle_{\mathfrak{n}}=0
$$


Thus $\langle\xi \cdot x, \eta\rangle=0$ for any $\eta \in \mathfrak{k}$. Namely, $\xi \cdot x \in \mathfrak{k}^{\perp}$ for any $\xi \in \mathfrak{n}_{p}$. Hence (4-3) holds.

Furthermore, we have an exact sequence

$$
0 \longrightarrow \mathfrak{n}_{x} \stackrel{i}{\longrightarrow} \mathfrak{n}_{p} \stackrel{j}{\longrightarrow} \mathfrak{k}^{\perp} \longrightarrow 0
$$

where $i$ is the inclusion.

To prove this, it only remains to show that

$$
\operatorname{dim}\left(\mathfrak{n}_{p} / \mathfrak{n}_{x}\right)=\operatorname{dim}\left(\mathfrak{k}^{\perp}\right) .
$$

For this, let us suppose $X$ is the coadjoint orbit of $G$ through $x$. As a subgroup of $G, N$ acts on $X$ in the Hamiltonian fashion. The moment map is

$$
\pi: X \rightarrow \mathfrak{n}^{*}, \quad \pi\left(x_{1}\right)=\left.x_{1}\right|_{\mathfrak{n}} .
$$

Let $\mathscr{O}$ be the $G$ orbit in $\mathfrak{n}^{*}$ through $p$. Then $\pi$ is a submersion from $X$ onto 0 .

It's a basic result of symplectic geometry that $\operatorname{Im}\left(d \pi_{x}\right)=\mathfrak{n}_{x}^{\perp}$ where $\mathfrak{n}_{x}^{\perp}$ is the annihilator of $\mathfrak{n}_{x}$ in $\mathfrak{n}^{*}$.

Hence,

$$
\operatorname{dim}(\mathscr{O})=\operatorname{dim}\left(\operatorname{Im}\left(d \pi_{x}\right)\right)=\operatorname{dim}\left(\mathfrak{n}_{x}^{\perp}\right)=\operatorname{dim}(N)-\operatorname{dim}\left(N_{x}\right) .
$$

It follows that

$$
\operatorname{dim}\left(N_{x}\right)=\operatorname{dim}(N)-\operatorname{dim}(\mathscr{O})=\operatorname{dim}(N)-\operatorname{dim}\left(G / G_{p}\right) .
$$

On the other hand, $Y \cong N / N_{p} \cong K / G_{p}$. So

$$
\operatorname{dim}(N)-\operatorname{dim}\left(N_{p}\right)=\operatorname{dim}(K)-\operatorname{dim}\left(G_{p}\right),
$$

or

$$
\operatorname{dim}(N)+\operatorname{dim}\left(G_{p}\right)=\operatorname{dim}\left(N_{p}\right)+\operatorname{dim}(K) .
$$

By (4-9) and (4-10),

$$
\begin{aligned}
\operatorname{dim}\left(N_{x}\right) & =\operatorname{dim}(N)-\left(\operatorname{dim}(G)-\operatorname{dim}\left(G_{p}\right)\right) \\
& =\operatorname{dim}(N)+\operatorname{dim}\left(G_{p}\right)-\operatorname{dim}(G) \\
& =\operatorname{dim}\left(N_{p}\right)-(\operatorname{dim}(G)-\operatorname{dim}(K)) \\
& =\operatorname{dim}\left(N_{p}\right)-\operatorname{dim}\left(\mathfrak{k}^{\perp}\right) .
\end{aligned}
$$

Namely,

$$
\operatorname{dim}\left(N_{p} / N_{x}\right)=\operatorname{dim}\left(\mathfrak{k}^{\perp}\right) .
$$

This completes the proof of (4-5).

We now go to the group level via the exponential map. Note that

$$
\exp (\xi) \cdot x=x+\xi \cdot x+\frac{1}{2 !} \xi^{2} \cdot x+\cdots
$$

for any $\xi \in \mathfrak{n}_{p}$.

One may observe that for any $x_{1} \in \mathfrak{k}^{\perp}$,

$$
\xi \cdot x_{1}=0 \text {. }
$$

Indeed, for any $\eta \in \mathfrak{g}$,

$$
[\xi, \eta] \in \mathfrak{n} \subseteq \mathfrak{k} .
$$


So

$$
\left\langle x_{1},[\xi, \eta]\right\rangle=0 .
$$

This implies

$$
\left\langle\xi \cdot x_{1}, \eta\right\rangle=0
$$

for all $\eta \in \mathfrak{g}$. Hence (4-12) holds. Therefore, (4-11) is simplified to

$$
\exp (\xi) \cdot x=x+\xi \cdot x \in x+\mathfrak{k}^{\perp} \text {. }
$$

Since $N_{p}^{0}$ is connected, any $v \in N_{p}^{0}$ can be written as

$$
v=\exp \left(\xi_{1}\right) \cdots \exp \left(\xi_{l}\right)
$$

for some $\xi_{1}, \cdots, \xi_{l} \in \mathfrak{n}_{p}$. Then by (4-13) and (4-11), we have

$$
v \cdot x=x+x_{1}+\cdots+x_{l}
$$

where $x_{i}=\xi_{i} \cdot x \in \mathfrak{k}^{\perp}$.

Then (4-14) gives us an injection from $N_{p}^{0} \cdot x$ into $x+\mathfrak{k}^{\perp}$. We claim that it is also surjective since for any $y \in \mathfrak{k}^{\perp}$, we have by (4-5) an element $\xi \in \mathfrak{n}_{p}$ such that $\xi \cdot x=y$. Then $\exp (\xi) \cdot x=x+y$. This completes the proof of the lemma.

In this section we will assume that $N_{p}$ is connected and, for the moment, $G=K$. Hence $N_{p} \cdot x=x$, and $\phi^{\xi}$ is well defined.

We want to show that

$$
l\left(\xi^{\sharp}\right) \Omega_{Y}=d \phi^{\xi} .
$$

Note that $Y$ is an $N$-homogeneous space. Any vector at $q \in Y$ can be written as $\delta^{\sharp}(q)$ for some $\delta \in \mathfrak{n}$.

We need to show that

$$
l\left(\xi^{\sharp}\right) \Omega_{Y}\left(\delta^{\sharp}\right)=d \phi^{\xi}\left(\delta^{\sharp}\right) .
$$

Let $q=v p_{0} \in Y$, at $q$,

$$
\text { RHS of } \begin{aligned}
(4-16) & =\delta^{\sharp}\left(\phi^{\xi}\right)(q)=\left.\frac{d}{d t}\right|_{t=0} \phi^{\xi}(\exp (-t \delta) \cdot q) \\
& =\left.\frac{d}{d t}\right|_{t=0}\left\langle\exp (-t \delta) \cdot v x_{0}, \xi\right\rangle_{\mathfrak{g}} \\
& =-\left\langle\delta \cdot v x_{0}, \xi\right\rangle_{\mathfrak{g}}=\left\langle v x_{0},[\delta, \xi]_{\mathfrak{g}}\right\rangle_{\mathfrak{g}} \\
& =\left\langle x_{0}, v^{-1}[\delta, \xi]\right\rangle_{\mathfrak{g}}=\left\langle p_{0}, v^{-1}[\delta, \xi]\right\rangle_{\mathfrak{n}} \\
& =\langle q,[\delta, \xi]\rangle_{\mathfrak{n}}=-\langle q,[\xi, \delta]\rangle
\end{aligned}
$$

On the other hand, there exists a $\delta_{\xi} \in \mathfrak{n}$ such that $\delta_{\xi}^{\sharp}(q)=\xi^{\sharp}(q)$. Thus

$$
\text { LHS of }(4-16)=\Omega_{Y}\left(\delta_{\xi}^{\sharp}(q), \delta^{\sharp}(q)\right)=-\left\langle q,\left[\delta_{\xi}, \delta\right]\right\rangle \text {. }
$$

But $\delta_{\xi}^{\sharp}(q)=\xi^{\sharp}(q)$ means $\xi \cdot q=\delta_{\xi} \cdot q$ on $\mathfrak{n}$, so

$$
\langle\xi \cdot q, \delta\rangle=\left\langle\delta_{\xi} \cdot q, \delta\right\rangle \text {. }
$$

Namely,

$$
\langle q,[\xi, \delta]\rangle=\left\langle q,\left[\delta_{\xi}, \delta\right]\right\rangle
$$


Hence $(4-17)=(4-18)$, which says that $(4-16)$ holds.

Therefore, the $G$-action on $Y$ has an infinitesimal lift $\phi: \xi \longmapsto \phi^{\xi}$. In general, $\phi$ is not a Lie algebra homomorphism. There is a 2-cocycle $\sigma: \mathfrak{g} \times$ $\mathfrak{g} \longrightarrow \mathbb{R}$ satisfying

$$
\sigma(\xi, \eta)=-\sigma(\eta, \xi)
$$

and

$$
\sigma([\xi, \eta], \zeta)+\sigma([\eta, \zeta], \xi)+\sigma([\zeta, \xi], \eta)=0
$$

such that

$$
\left\{\phi^{\xi}, \phi^{\eta}\right\}=\sigma(\xi, \eta)+\phi^{[\xi, \eta]} .
$$

This 2-cocycle is just the Mackey 2-cocycle at the orbit level. It prevents $Y$ from being a coadjoint orbit of $G$. However, $Y$ is a coadjoint orbit of some central extension of $G$ with respect to the circle group $T$. At the algebra level, define

$$
\mathfrak{g}^{\sigma}=\{(s, \xi), s \in \mathbb{R}, \xi \in \mathfrak{g}\},
$$

where

$$
[(s, \xi),(t, \eta)]=\left(\sigma(\xi, \eta),[\xi, \eta]_{\mathfrak{g}}\right) .
$$

It is easy to check that (4-22) does define a Lie bracket for $\mathfrak{g}^{\sigma}$.

We have an exact sequence of Lie algebras

$$
0 \rightarrow \mathbb{R} \stackrel{i}{\longrightarrow} \mathfrak{g}^{\sigma} \stackrel{j}{\rightarrow} \mathfrak{g} \rightarrow 0,
$$

where $i(s)=(s, 0)$, and $j(s, \xi)=\xi . \mathfrak{g}^{\sigma}$ is a central extension of $\mathfrak{g}$ with respect to $\mathbb{R}$.

Let $G^{\sigma}$ be the corresponding central extension of $G$ with Lie algebra $\mathfrak{g}^{\sigma}$, we have

$$
1 \rightarrow T \stackrel{I}{\longrightarrow} G^{\sigma} \stackrel{J}{\longrightarrow} G \rightarrow e .
$$

$G^{\sigma}$ acts on $Y$ by

$$
\tilde{a} \cdot q=J(\tilde{a}) \cdot q, \quad \text { for any } \tilde{a} \in G^{\sigma}, q \in Y .
$$

The corresponding infinitesimal action is $(s, \xi) \cdot q=\xi \cdot q$. Define

$$
\psi^{(s, \xi)}: Y \longrightarrow \mathbb{R}, \quad \psi^{(s, \xi)}\left(v p_{0}\right)=\left\langle v x_{0}, \xi\right\rangle+s .
$$

Since $(s, \xi)^{\sharp}=\xi^{\sharp}$, and $\psi^{(s, \xi)}=\phi^{\xi}+s$, we have

$$
\imath\left((s, \xi)^{\sharp}\right) \Omega_{Y}=d \psi^{(s, \xi)} .
$$

So $\psi^{(s, \xi)}$ is a lift of the action of $(s, \xi)$. Furthermore,

$$
\left\{\psi^{(s, \xi)}, \psi^{(t, \eta)}\right\}=\left\{\phi^{\xi}+s, \phi^{\eta}+t\right\}=\left\{\phi^{\xi}, \phi^{\eta}\right\}=\sigma(\xi, \eta)+\phi^{[\xi, \eta]} .
$$

But

$$
\psi^{[(s, \xi),(t, \eta)]}=\psi^{(\sigma(\xi, \eta),[\xi, \eta])}=\sigma(\xi, \eta)+\phi^{[\xi, \eta]} .
$$

Hence,

$$
\left\{\psi^{(s, \xi)}, \psi^{(t, \eta)}\right\}=\psi^{[(s, \xi),(t, \eta)]} .
$$


This implies that $\psi:(s, \xi) \longmapsto \psi^{(s, \xi)}$ is a Lie algebra homomorphism.

Therefore, $\psi$ defines a moment map $\Psi$ for the $G^{\sigma}$-action on $Y$; in other words, the $G^{\sigma}$-action on $Y$ is Hamiltonian. Moreover, $\Psi\left(v p_{0}\right) \in\left(\mathfrak{g}^{\sigma}\right)^{*}$, and

$$
\left\langle\Psi\left(v p_{0}\right),(s, \xi)\right\rangle=\psi^{(s, \xi)}\left(v p_{0}\right)=\left\langle v x_{0}, \xi\right\rangle+s,
$$

so

$$
\Psi\left(v p_{0}\right)=v x_{0}+1^{*}, \quad \text { where } 1^{*} \in \mathbb{R}^{*}, 1^{*}(s)=s .
$$

If $v_{1} x_{0}=v_{2} x_{0}$, then $v_{1} p_{0}=v_{2} p_{0}$. Thus $\Psi$ is one-one. By Theorem 3.2, $Y$ is a coadjoint orbit of $G^{\sigma}$.

To get a coadjoint orbit of $G$, we need to get rid of the 2-cocycle $\sigma$. For this purpose, we consider another $G$-homogeneous symplectic space $\left(W, \Omega_{W}\right)$ satisfying:

(I) $N$ acts on $W$ trivially;

(II) $G$ acts on $W$ symplectically with the infinitesimal lifting which has the obstruction $-\sigma$.

Before discussing the existence of such a $\left(W, \Omega_{W}\right)$, let us see how it works first.

We form the product space $Y \times W$ with the product symplectic form. It is easy to see that the first condition implies

Lemma 4.2. $G$ acts on $Y \times W$ transitively.

Proof. For any $\left(p_{1}, w_{1}\right)$ and $\left(p_{2}, w_{2}\right) \in Y \times W$, we may find an element $a \in G$ such that $a \cdot w_{1}=w_{2}$. On the other hand, $a p_{1}$ and $p_{2}$ are in $Y$, hence we have an element $v \in N$ such that $v \cdot a p_{1}=p_{2}$. But $N$ acts on $Y$ trivially. Hence $v a \cdot\left(p_{1}, w_{1}\right)=\left(p_{2}, w_{2}\right)$.

The second condition implies that $W$ is a coadjoint orbit of the group extension $G^{-\sigma}$. When $Y$ and $W$ product together, the obstructions cancel out. Therefore we have

Proposition 4.2. $Y \times W$ is a covering space of some coadjoint orbit of $G$.

Proof. Let us denote by $\phi_{1}^{\xi}$ the lift of the infinitesimal action of $\xi$ on $W$. Set

$$
\psi_{1}: \mathfrak{g} \longrightarrow \operatorname{Poisson}(Y \times W),
$$

$\psi_{1}(\xi)=\psi_{1}^{\xi}$, where

$$
\psi_{1}^{\xi}(p, w)=\phi^{\xi}(p)+\phi_{1}^{\xi}(w) .
$$

Then $\xi \longmapsto \psi_{1}^{\xi}$ is a lift of the infinitesimal action of $\xi$ on $Y \times W$. Moreover,

$$
\begin{aligned}
\left\{\psi_{1}^{\xi}, \psi_{1}^{\eta}\right\}(p, w) & =\left\{\phi^{\xi}, \phi^{\eta}\right\}(p)+\left\{\phi_{1}^{\xi}, \phi_{1}^{\eta}\right\}(w) \\
& =\sigma(\xi, \eta)+\phi^{[\xi, \eta]}-\sigma(\xi, \eta)+\phi_{1}^{[\xi, \eta]} \\
& =\psi_{1}^{[\xi, \eta]} .
\end{aligned}
$$

Hence $\psi_{1}$ is a Lie algebra homomorphism. Therefore, it gives rise to a moment map $\Psi_{1}: Y \times W \longrightarrow \mathfrak{g}^{*}$. By Theorem 3.2, $Y \times W$ is a covering of some coadjoint orbit of $G$.

If we can choose $W$ good enough so that the moment map $\Psi_{1}$ is one-one, we then get a coadjoint orbit of $G$ from $Y$. As matter of fact, such a "good" $W$ always exists. 
Let us discuss this in a little bit more generality. We consider now the situation of Lemma 4.1; namely, we no longer assume $G$ fixes $Y$ and consider the stabilizer subgroup $K=G_{Y}$. The problem now is to get a coadjoint orbit of $K$ from $Y$. We take a coadjoint orbit $X$ of $G$ sitting over $Y$ in the sense that $\left.X\right|_{\mathfrak{n}} \supseteq Y$. Let

$$
\pi: X \longrightarrow \mathrm{n}^{*}
$$

be the projection. It is actually the moment map for the $N$-action on $X$. Denote by $Y^{-}$the space $Y$ equipped with the negative symplectic form $-\Omega_{Y}$, let

$$
\pi_{1}: X \times Y^{-} \longrightarrow \mathfrak{n}^{*}
$$

be the map $\pi_{1}(x, p)=\pi(x)-p$. It is the moment map for the product action of $N$ on $X \times Y^{-}$with 0 as a regular value. Let $W$ be the orbit space $\pi_{1}^{-1}(0) / N$. To see the space $W$ is well defined, we need the following lemma.

Lemma 4.3. Under the assumption of Lemma 4.1, $G_{x} N_{p}$ is a closed subgroup of $G$.

Proof. First of all, since $G_{x}$ stabilizes $N_{p}, G_{x} N_{p}$ is a subgroup. To show that $G_{x} N_{p}$ is closed, we only need to show that $N_{p} G_{x}$ is closed. We claim

$$
N_{p} G_{x}=\left\{a \in G \mid a x \in x+\mathfrak{k}^{0}\right\} .
$$

Indeed, by Lemma 4.1, it is obvious that

$$
N_{p} G_{x} \subseteq\left\{a \in G \mid a x \in x+\mathfrak{k}^{0}\right\} .
$$

On the other hand, for any $a \in\left\{a \in G \mid a x \in x+\mathfrak{k}^{0}\right\}$, by Lemma 4.1, there exists an element $v \in N_{p}$ such that $a x=v x$. This implies $v^{-1} a \in G_{x}$. Namely, $a=v b$ for some $b \in G_{x}$. This concludes the proof.

Corollary 4.1. The space $\pi^{-1}(p) / N_{p}$ is a well-defined manifold.

Proof. In fact, $\pi^{-1}(p)=K_{p} / G_{x}$, so

$$
\pi^{-1}(p) / N_{p}=K_{p} / G_{x} N_{p} .
$$

By Lemma 4.3, $G_{x} N_{p}$ is closed. Hence $\pi^{-1}(p)$ is well defined.

In terms of the Marsden-Weinstein reduction, we get a reduced space $\pi^{-1}(p) / N_{p}$. Guillemin's version of this symplectic manifold is just $W=$ $\pi_{1}^{-1}(0) / N$. Therefore, $W$ is a well-defined symplectic manifold. Since $N \subset K$ is normal, we can define a $K$-action on $W$ as follows:

$$
a \cdot[x, p]=[a x, a p], \quad a \in K,[x, p] \in W .
$$

The $K$-actions on $X$ and $Y^{-}$are symplectic. It follows that the $K$-action on $X \times Y^{-}$is symplectic. The latter action induces a symplectic $K$-action on $W$.

We have the infinitesimal lifting $\phi: \mathfrak{k} \longrightarrow$ Poisson $(Y)$. Let us now define a map

$$
\phi_{1}: \mathfrak{k} \longrightarrow \operatorname{Poisson}(W),
$$

$\phi_{1}(\xi)=\phi_{1}^{\xi}$, where

$$
\phi_{1}^{\xi}([x, p])=\langle\xi, x\rangle_{\mathfrak{g}}-\phi^{\xi}(p)
$$

for any $[x, p] \in W$. 
To see that $\phi_{1}$ is well defined, we only need to check that

$$
\phi_{1}^{\xi}\left(\left[x, p_{0}\right]\right)=\phi_{1}^{\xi}\left(\left[x_{1}, p_{0}\right]\right)
$$

for $x, x_{1} \in X,\left.x\right|_{\mathfrak{n}}=p_{0},\left.\quad x_{1}\right|_{\mathfrak{n}}=p_{0}$ and $\left[x, p_{0}\right]=\left[x_{1}, p_{0}\right] \in W$.

But $\left[x, p_{0}\right]=\left[x_{1}, p_{0}\right]$ implies that there exists $u \in N_{p_{0}}$ such that $x_{1}=u x$. Hence

$$
\begin{aligned}
\phi_{1}^{\xi}\left(\left[x_{1}, p_{0}\right]\right) & =\left\langle\xi, x_{1}\right\rangle_{\mathfrak{g}}-\phi^{\xi}\left(p_{0}\right)=\left\langle\xi,\left.x_{1}\right|_{\mathfrak{k}}\right\rangle_{\mathfrak{k}}-\phi^{\xi}\left(p_{0}\right) \\
& =\left\langle\xi,\left.(u x)\right|_{\mathfrak{k}}\right\rangle_{\mathfrak{k}}-\phi^{\xi}\left(p_{0}\right)=\left\langle\xi, u\left(\left.x\right|_{\mathfrak{k}}\right)\right\rangle_{\mathfrak{k}}-\phi^{\xi}\left(p_{0}\right) .
\end{aligned}
$$

By Lemma 4.1, since $N_{p_{0}}$ is connected, $N_{p_{0}}$ fixes $\left.x\right|_{\mathfrak{k}}$. Thus, we conclude

$$
\phi_{1}^{\xi}\left(\left[x_{1}, p_{0}\right]\right)=\left\langle\xi,\left.x\right|_{\mathfrak{k}}\right\rangle_{\mathfrak{k}}-\phi^{\xi}\left(p_{0}\right)=\phi_{1}^{\xi}\left(\left[x, p_{0}\right]\right) .
$$

Proposition 4.3. $\phi_{1}^{\xi}$ is the lifting of the infinitesimal action of $\xi$ on $W$ with the obstruction $-\sigma$.

Proof. Let

$$
\phi_{2}^{\xi}: X \longrightarrow \mathbb{R}, \quad \phi_{2}^{\xi}(x)=\langle\xi, x\rangle_{\mathfrak{g}} .
$$

It is well known that $\phi_{2}^{\xi}$ is the lift of the infinitesimal $K$-action on $X$, which is Hamiltonian. There is no obstruction for $\phi_{2}^{\xi}$.

First, we show that

$$
l\left(\xi_{W}^{\sharp}\right) \Omega_{W}=d \phi_{1}^{\xi} .
$$

For any $\Xi \in \mathscr{X}(W)$, a vector field on $W$, there exists a vector field $\Xi_{1} \in$ $\mathscr{X}\left(\pi_{1}^{-1}(0)\right) \subset \mathscr{X}\left(X \times Y^{-}\right)$such that $j_{1 *}\left(\Xi_{1}\right)=\Xi$, where $j_{1}: \pi_{1}^{-1}(0) \longrightarrow W$ is the projection. We denote by $i_{1}$ the inclusion $\pi_{1}^{-1}(0) \longrightarrow X \times Y^{-}$. Now for any $(x, p) \in \pi_{1}^{-1}(0)$,

$$
\Xi_{1}(x, p)=\Xi_{1}^{\prime}(x) \oplus \Xi_{1}^{\prime \prime}(p),
$$

where $\Xi_{1}^{\prime}(x) \in T_{x} X$ and $\Xi_{1}^{\prime \prime}(p) \in T_{p} Y$. So

$$
\begin{aligned}
d \phi_{1}^{\xi}(\Xi)([x, p]) & =d \phi_{1}^{\xi}\left(j_{1 *}\left(\Xi_{1}\right)\right)([x, p])=j_{1 *} d \phi_{1}^{\xi}\left(\Xi_{1}\right)(x, p) \\
& =d\left(j_{1}^{*} \phi_{1}^{\xi}\right)\left(\Xi_{1}\right)(x, p)=\Xi_{1}\left(j_{1}^{*} \phi_{1}^{\xi}\right)(x, p) \\
& =\Xi_{1}^{\prime}\left(\phi_{2}^{\xi}\right)(x)-\Xi_{1}^{\prime \prime}\left(\phi^{\xi}\right)(p)=d \phi_{2}^{\xi}\left(\Xi_{1}^{\prime}\right)(x)-d \phi^{\xi}\left(\Xi_{1}^{\prime \prime}\right)(p) .
\end{aligned}
$$

On the other hand,

$$
\begin{aligned}
l\left(\xi_{W}^{\sharp}\right) \Omega_{W}(\Xi)([x, p]) & =\Omega_{W}\left(\xi_{W}^{\sharp}, \Xi\right)([x, p]) \\
& =\Omega_{W}\left(j_{1 *} \xi_{X \times Y^{-}}^{\sharp}, \Xi_{1}\right)([x, p]) \\
& =i^{*} \Omega_{X \times Y^{-}}\left(\xi_{X \times Y^{-}}^{\sharp}, \Xi_{1}\right)(x, p) \\
& =\Omega_{X}\left(\xi_{X}^{\sharp}, \Xi_{1}^{\prime}\right)(x)-\Omega_{Y}\left(\xi_{Y}^{\sharp}, \Xi_{1}^{\prime \prime}\right)(p) .
\end{aligned}
$$

Comparing (4-35) and (4-36) we see that (4-34) holds.

To compute the obstruction,

$$
\begin{aligned}
\left\{\phi_{1}^{\xi}, \phi_{1}^{\eta}\right\}([x, p]) & =\left\{\phi_{2}^{\xi}, \phi_{2}^{\eta}\right\}(x)-\left\{\phi^{\xi}, \phi^{\eta}\right\}(p) \\
& =\phi_{2}^{[\xi, \eta]}(x)-\phi^{[\xi, \eta]}(p)-\sigma(\xi, \eta) \\
& =\phi_{1}^{[\xi, \eta]}([x, p])-\sigma(\xi, \eta) .
\end{aligned}
$$


Therefore, the obstruction for $\phi_{1}$ is $-\sigma$.

Corollary 4.2. $W$ is a coadjoint orbit of the central extension $K^{-\sigma}$ of $K$ with respect to $T$.

Our $W$ satisfies the two conditions we set before. Also, we have

Lemma 4.4. $K$ acts on $W$ transitively.

Proof. For any two points $\left[x_{1}, p_{0}\right],\left[x_{2}, p_{0}\right] \in W$, we may find $g \in G$ such that $g \cdot x_{1}=x_{2}$. It follows that $g \cdot p_{0}=p_{0}$ so that $g \in K$. But then $a \cdot\left[x_{1}, p_{0}\right]=\left[x_{2}, p_{0}\right]$.

We now have the material we need to get a coadjoint orbit of $K$.

Proposition 4.4. $Y \times W$ is a coadjoint orbit of $K$.

Proof. It only remains to show that the moment map

$$
\begin{gathered}
\Psi_{1}: Y \times W \longrightarrow \mathfrak{k}^{*}, \\
\Psi_{1}\left(v p_{0},\left[x, p_{0}\right]\right)=v x_{0}+\left.x\right|_{\mathfrak{k}}-x_{0},
\end{gathered}
$$

where $x_{0}$ is a point in $\mathfrak{k}^{*}$ sitting over $p_{0} \in Y$ is one-one.

We claim that this moment map can be rewritten as

$$
\Psi_{1}\left(v p_{0},\left[x, p_{0}\right]\right)=\left.v x\right|_{\mathfrak{k}} .
$$

To see this, we need to show

$$
v x_{0}+\left.x\right|_{\mathfrak{k}}-x_{0}=\left.v x\right|_{\mathfrak{k}} .
$$

In other words,

$$
v\left(\left.x\right|_{\mathfrak{k}}-x_{0}\right)=\left.x\right|_{\mathfrak{k}}-x_{0}
$$

for any $v \in N$.

Note that

$$
\left.\left(\left.x\right|_{\mathfrak{k}}-x_{0}\right)\right|_{\mathfrak{n}}=p_{0}-p_{0}=0 .
$$

In fact, we claim that for any $w \in \mathfrak{k}^{*},\left.w\right|_{\mathfrak{n}}=0$, then $v \cdot w=w$ on $\mathfrak{k}$. Since $N$ is connected, we only need to consider it at the algebra level. For any $\delta \in \mathfrak{n}, \quad \eta \in \mathfrak{k}$,

$$
\langle\delta \cdot w, \eta\rangle_{\mathfrak{k}}=-\langle w,[\delta, \eta]\rangle=0
$$

since for $[\delta, \eta] \in \mathfrak{n}$.

Hence $\delta \cdot w=0$.

We now show that this moment map is one-one. Suppose $\left.v_{1} x_{1}\right|_{\mathfrak{t}}=\left.v_{2} x_{2}\right|_{\mathfrak{t}}$, then $v_{1} p_{0}=v_{2} p_{0}$ since for $x_{1}$ and $x_{2}$ all sit over $p_{0}$. It follows that $v_{2}^{-1} v_{1} \in$ $N_{p_{0}}$. Since $\left.v_{2}^{-1} v_{1} x_{1}\right|_{\mathfrak{k}}=\left.x_{2}\right|_{\mathfrak{k}}$, by Lemma 4.1 there exists an element $v \in N_{p_{0}}$ such that $v v_{2}^{-1} v_{1} x_{1}=x_{2}$. Let $u=v v_{2}^{-1} v_{1} \in N_{p_{0}}$, we conclude that

$$
\left(v_{1} p_{0},\left[x_{1}, p_{0}\right]\right)=\left(v_{2} p_{0},\left[u x_{1}, u p_{0}\right]\right)=\left(v_{2} p_{0},\left[x_{2}, p_{0}\right]\right) .
$$

So $\Psi_{1}$ is 1-1. By Theorem 3.2, $Y \times W$ is a coadjoint orbit of $K$.

Remark 4.1. We show elsewhere that all coadjoint orbits of $G$ can be constructed from the "little group data" $Y \times W$ by the techniques of symplectic induction (see [6]). 


\section{REFERENCES}

1. L. Auslander and B. Kostant, Polarization and unitary representations of solvable Lie groups, Invent. Math. 14 (1971), 255-354.

2. J. Brezin, Unitary representation theory for solvable Lie groups, Mem. Amer. Math. Soc. No. 79 (1968).

3. R. Bryant, An introduction to Lie groups and symplectic geometry, Preprint, Lecture Notes in RGI, Park City, Utah, 1991.

4. V. W. Guillemin and S. Sternberg, Symplectic techniques in physics, Cambridge Univ. Press, 1984.

5. A. A. Kirillov, Unitary representations of nilpotent Lie groups, Uspehi Mat. Nauk 17 (1962), 57-110.

6. N. Kostant, Quantization and unitary representations, Lecture Notes in Math., vol. 170, Springer-Verlag, New York, 1970, pp. 87-207.

7. Z. Li, Coadjoint orbits and induced representations, Ph.D. Thesis, MIT, 1993.

8. G. W. Mackey, Induced representations of locally compact groups, I, Ann. of Math. (2) 55 (1952), 101-139.

9. G. W. Mackey, Unitary representations of group extensions, I, Acta Math. 99 (1958), 265-311.

10. _ The theory of unitary group representations, Univ. of Chicago Press, Chicago, 1976.

Department of Mathematics, Florida International University, Miami, Florida 33199

E-mail address: lizongyi@fiu.edu 